\title{
Factores predictivos de conversión de colecistectomía laparoscópica a abierta en pacientes de una clínica privada, 2017
}

\author{
Predictive factors for conversion from laparoscopic to open cholecystectomy in patients in a private \\ clinic, 2017 \\ Emilio Contreras Alomía ${ }^{1 *}$, Flor Contreras Castro
}

\begin{abstract}
RESUMEN
Objetivo: el estudio se desarrolló con el objetivo de determinar los factores predictivos de conversión de cirugía laparoscópica (CL) a cirugía abierta (CA) en una clínica privada. Materiales y Métodos: El estudio fue retrospectivo basado en la revisión de las historias clínicas de todos los pacientes sometidos a CL haciendo un total de 255 casos entre enero de 2012 y diciembre de 2016. Resultados: Los resultados mostraron una tasa de conversión de $11.4 \%$; el análisis de regresión logística reportó como mejores factores predictivos al diagnóstico de colecistitis aguda $(\mathrm{OR}=79.384, \mathrm{p}=0.000)$, sexo $(\mathrm{OR}=6.246, \mathrm{p}=0.002)$ y edad $(\mathrm{OR}=1.038, \mathrm{p}=0.038)$. Conclusiones: Aunque existen otras variables que tienen relación significativa con la conversión de la cirugía, el análisis estadístico muestra estas tres variables como predictores.
\end{abstract}

Palabras clave: Colecistectomía, cirugía laparoscópica, conversión de cirugía, cirugía abierta.

\begin{abstract}
Objective: The aim of the study was to determine the predictive factors of conversion from laparoscopic surgery (CL) to open surgery in a private clinic: Materials and Methods: the study was retrospective based on the review of the clinical records of All patients undergoing CL totaling 255 cases between January 2012 and December 2016. Results: Results showed a conversion rate of $11.4 \%$; $(\mathrm{OR}=79.384, \mathrm{p}=0.000)$, sex $(\mathrm{OR}=6,246, \mathrm{p}=0.002)$ and age $(\mathrm{OR}=1,038, \mathrm{p}=0.038)$ were the best predictors of the diagnosis of acute cholecystitis. Conclusions: Although there are other variables that have a significant relationship with the conversion of surgery, the statistical analysis shows these three variables as predictors.
\end{abstract}

Keywords: Cholecystectomy, laparoscopic surgery, Surgery conversión, Open surgery.

${ }^{1}$ Jefe del servicio de cirugía de Clínica Adventista Ana Stahl. Iquitos, Perú.

${ }^{2}$ Docente de EP Enfermería. Facultad de Ciencias de la Salud. Universidad Peruana Unión, Lima Perú. 


\section{INTRODUCCIÓN}

Debido a la alta incidencia de la litiasis vesicular, esta patología en su forma aguda representa la segunda causa de abdomen agudo en el mundo (Ibáñez et al., 2007; Vargas et al., 2017). Aproximadamente entre el $10-15 \%$ de la población en los países desarrollados presenta litiasis biliar (Domínguez, Rivera, Bermúdez, \& Herrera, 2011; Serafini, 2016). La colecistectomía laparoscópica se constituye actualmente en el gold estándar para el tratamiento de la litiasis vesicular sintomática y ha sustituido a la colecistectomía abierta o convencional en forma paulatina desde su introducción a inicios de la década de los 90 del siglo pasado (Bueno-Lledó et al., 2005; Campos \& Lara, 2012; J. Pekolj \& Mazza, 2005; Requena \& Gutiérrez, 2007; Simón, Sánchez, Acosta, \& Sosa, 2006).

Desde que en 1985 el alemán Erich Mühe realizó la primera CL, y en 1987 el francés Philippe Mouret hizo lo propio utilizando el videolaparoscopio, la CL se extendió por todo el orbe (Borie \& Millat, 2003; Ramos, Álvarez, Lorenzo, Vallés, \& Gallardo, 2014; Serafini, 2016). Es así como el 13 de febrero de 1991 en el Hospital Nacional Edgardo Rebagliati Martins de la ciudad de Lima en Perú, se lleva a cabo el primer procedimiento de este tipo por Alfredo Sepúlveda (Sepulveda, 1991).

Actualmente, la CL una de las intervenciones quirúrgicas que más se llevan a cabo en los centros hospitalarios públicos y privados a nivel mundial (Galloso, Frías, Pérez, Petersson, \& Benavides, 2012). Siendo que su reproducción es constante y frecuente, existen condiciones o factores que influyen en la conversión a CA y que clásicamente son: el sexo, edad, obesidad, cirugía abdominal previa, enfermedades subyacentes, patología aguda, entre otros (Galloso et al., 2012; Priego et al., 2008; Ramos et al., 2014). La tasa de conversión a nivel mundial es variada y oscila entre el $2-15 \%$ mientras que en América Latina el rango va del 0.8-20\% (Álvarez et al., 2013; Balagué et al., 2000; Bocanegra \& Córdova, 2013; Bueno et al., 2001; Domínguez, Rivera, et al., 2011; Lucema, Coronel, \& Orellana, 2008; Paredes et al., 2001). Dentro de las causas de conversión puede citarse la inflamación severa que dificulta la adecuada visualización de las estructuras anatómicas que forman el triángulo de Calot, anatomía no precisa, el sangrado, patología adherencial y la lesión de la vía biliar (Ibáñez et al., 2007; Juan Pekolj \& Sánchez, 2015; Serafini, 2016; Vargas et al., 2017).

El objetivo del presente estudio es determinar los factores predictivos de conversión de CL a CA en base a la experiencia de la Clínica Adventista Ana Sthal de
Iquitos; dado que, a nivel regional, en el departamento de Loreto (Amazonia peruana), no se dispone de datos ni estudios formales que ilustren lo planteado, y a nivel nacional los estudios son escasos al respecto (Bocanegra \& Córdova, 2013).

\section{MATERIAL Y MÉTODOS}

Se realizó un análisis retrospectivo basado en la revisión de las historias clínicas de todos los pacientes sometidos a colecistectomía laparoscópica (CL) haciendo un total de 255 casos entre enero de 2012 y diciembre de 2016 en la Clínica Adventista Ana Sthal de la ciudad de Iquitos, departamento de Loreto, Perú. Las CL fueron realizadas por cirujanos con experiencia en técnica estándar de cuatro puertos (Técnica Americana y Técnica Francesa).

Se registraron los datos de las variables edad, género, diagnóstico preoperatorio, cirugía programada o de urgencia, así como la causa de la conversión de cada caso en una ficha de recolección estructurada por el investigador. Los datos fueron recolectados considerando los horarios de menor confluencia de usuarios a fin de no interferir con las actividades del personal que hace uso de las historias clínicas. Para el tratamiento de los datos se utilizó el software SPSS versión 24, donde fueron ingresados los datos de cada caso, aplicando la opción de recodificación para asignar los valores adecuados a las variables sexo (0: femenino, 1: masculino), tipo de atención de colecistectomía (0: programada, 1: urgencia), antecedente de cirugía ( 0 : no, 1: si) y conversión de cirugía (0: no, 1: si). Se utilizó esta misma opción para dicotomizar los diagnósticos preoperatorios (colecistitis aguda, colelitiasis y poliposis vesicular) y motivo de la conversión (Inflamación severa, adherencias y sangrado); el valor " 0 " etiquetado como no, representa a todos los casos que no presentaron el diagnóstico o motivo de conversión, y el valor "1" etiquetado como si, a los casos que si lo presentaron.

Se realizó el análisis bivariado para estimar la asociación de los indicadores considerados en la conversión de la cirugía. Para las variables cualitativas, se aplicó la prueba X2, y para aquellas donde no se cumplieron los supuestos, se procedió a realizar el cálculo del Test exacto de Fisher; para la variable edad, se utilizó la prueba $\mathrm{T}$ de Student. Los descriptivos se presentaron utilizando porcentajes para las variables cualitativas, $\mathrm{y}$ la media acompañado de las medidas de variabilidad para la edad. Posteriormente, se realizó el análisis multivariado previa evaluación de las condiciones y adecuación de los datos; el método utilizado fue pasos de Wald hacia atrás. Se eligió el séptimo modelo por presentar mayor significancia estadística. 
Las consideraciones éticas que se tomaron en cuenta fueron la confidencialidad mediante el anonimato de los pacientes, integridad de los datos recopilados a través del cumplimiento de los estándares propuestos para el tratamiento estadístico adecuado de los mismos, y la responsabilidad que está íntimamente ligada con la no maleficencia al publicar los datos.

\section{RESULTADOS}

Tabla 1

Análisis bivariado de las variables consideradas predictores de la conversión de cirugía.

\begin{tabular}{|c|c|c|c|}
\hline \multirow[t]{2}{*}{ Factores } & \multicolumn{2}{|c|}{ Conversión de cirugía } & \multirow[t]{2}{*}{ Estadístico $(p)$} \\
\hline & $\begin{array}{l}\text { No } \\
{[226(88.6 \%)]}\end{array}$ & $\begin{array}{l}\mathrm{Si} \\
{[29(11.4 \%)]}\end{array}$ & \\
\hline Cuantitativos & $\overline{\mathrm{x}} \pm S($ Min-Max $)$ & $\overline{\mathrm{X}} \pm S$ (Min-Max) & $t(\mathrm{p})$ \\
\hline Edad & $\begin{array}{l}39.74 \pm 13.47 \\
(11-77)\end{array}$ & $\begin{array}{l}54.52 \pm 19.69 \\
(24-89)\end{array}$ & $-3.924(0.000)$ \\
\hline Cualitativos & $\mathrm{n}(\%)$ & $n(\%)$ & $\begin{array}{l}X^{2}(p) \\
{[\text { Corrección }]}\end{array}$ \\
\hline \multicolumn{4}{|l|}{ Sexo } \\
\hline Femenino & $177(69.4)$ & $11(4.3)$ & \multirow[t]{2}{*}{$21.643(0.000)$} \\
\hline Masculino & 49(19.2) & $18(7.1)$ & \\
\hline \multicolumn{4}{|c|}{ Antecedente de cirugía } \\
\hline No & $169(66.3)$ & $21(8.2)$ & \multirow[t]{2}{*}{$0.076(0.783)$} \\
\hline Si & $57(22.4)$ & $8(3.1)$ & \\
\hline \multicolumn{4}{|c|}{ Tipo de atención de colecistectomía } \\
\hline Programada & 192(75.3) & $2(0.8)$ & \multirow[t]{2}{*}{$86.052(0.000)$} \\
\hline Urgencia & $34(13.3)$ & $27(10.6)$ & \\
\hline \multicolumn{4}{|c|}{ Colecistitis aguda } \\
\hline No & $190(74.5)$ & $2(0.8)$ & \multirow[t]{2}{*}{$82.290(0.000)$} \\
\hline Si & $36(14.1)$ & $27(10.6)$ & \\
\hline \multicolumn{4}{|l|}{ Colelitiasis } \\
\hline No & $48(18.8)$ & $27(10.6)$ & \multirow[t]{2}{*}{$63.935(0.000)$} \\
\hline Si & $178(69.8)$ & $2(0.8)$ & \\
\hline \multicolumn{4}{|c|}{ Poliposis vesicular } \\
\hline No & $214(83.9)$ & $29(11.4)$ & \multirow{2}{*}{$\begin{array}{l}1.616(0.204)^{b} \\
{[0.371]}\end{array}$} \\
\hline Si & $12(4.7)$ & $0(0.0)$ & \\
\hline \multicolumn{4}{|c|}{ Inflamación severa } \\
\hline No & $226(88.6)$ & $5(2.0)$ & \multirow{2}{*}{$\begin{array}{l}206.467(0.000)^{b} \\
{[0.000]}\end{array}$} \\
\hline Si & $0(0.0)$ & $24(9.4)$ & \\
\hline \multicolumn{4}{|l|}{ Adherencias } \\
\hline No & $226(88.6)$ & $27(10.6)$ & \multirow{2}{*}{$\begin{array}{l}15.709(0.000)^{b, c} \\
{[0.013]}\end{array}$} \\
\hline Si & $0(0.0)$ & $2(0.8)$ & \\
\hline \multicolumn{4}{|l|}{ Sangrado } \\
\hline No & $226(88.6)$ & $27(10.6)$ & \multirow{2}{*}{$\begin{array}{l}15.709(0.000)^{b, c} \\
{[0.013]}\end{array}$} \\
\hline Si & $0(0.0)$ & $2(0.8)$ & \\
\hline
\end{tabular}

Nota: Evaluación de supuestos de la prueba chi cuadrado (b) Más del 20\% de las frecuencias esperadas son menores a 5; (c) la frecuencia esperada es menor a 1 . 
Los resultados de la tabla 1 muestran que, de los 255 registros de órdenes para $\mathrm{CL}$, la tasa de conversión a CA fue del $11.4 \%$; la edad de los pacientes atendidos con $\operatorname{CL}(\overline{\mathrm{X}}=39.74, \mathrm{~S}=13.47)$ fue menor en comparación al grupo que presentó conversión de cirugía $(\overline{\mathrm{X}}=54.52$, $\mathrm{S}=19.69$ ); la mayor cantidad de casos atendidos fueron del sexo femenino ( $\mathrm{CL}=69.4 \%, \mathrm{CA}=4.3 \%)$, con una tasa de conversión mayor evidenciada en los casos del sexo masculino $(\mathrm{CL}=19.2 \%, \mathrm{CA}=7.1 \%)$; los casos con antecedente de cirugía ( $\mathrm{CL}=22.4 \%, \mathrm{CA}=3.1 \%$ ) fueron menoresal grupoquenolotuvo $(\mathrm{CL}=66.3 \%, \mathrm{CA}=8.2 \%)$; la mayoría de las atenciones de colecistectomía fueron programadas $(\mathrm{CL}=75.3 \%, \mathrm{CA}=0.8 \%)$; $\sin$ embargo, hubo una cantidad considerable de casos atendidos de urgencia $(\mathrm{CL}=13.3 \%, \mathrm{CA}=10.6 \%)$. En cuanto al análisis por diagnóstico preoperatorio $\mathrm{y}$ motivo de la conversión, se presentan los siguientes resultados: la colelitiasis fue el diagnóstico mayoritario $(\mathrm{CL}=178[69.8 \%], \quad \mathrm{CA}=2[0.8 \%]), \quad$ seguido de la colecistitis aguda $(\mathrm{CL}=36[14.1 \%], \mathrm{CA}=27[10.6 \%])$, y la poliposis vesicular $(\mathrm{CL}=12[4.7 \%], \mathrm{CA}=0[0.0 \%])$. El motivo de la conversión con mayor registro fue la inflamación severa $(\mathrm{CA}=24[9.4 \%])$, seguido de las adherencias y el sangrado con porcentajes iguales $(\mathrm{CA}=2[0.8 \%])$. Evidencian relación con la conversión a cirugía abierta con un $\alpha=0.01$ : la edad $(\mathrm{t}=-3.924$, $\mathrm{p}=0.000)$, el sexo $\left(\mathrm{X}^{2}=21.643, \mathrm{p}=0.000\right)$, el tipo de atención de la colecistectomía $\left(\mathrm{X}^{2}=86.052, \mathrm{p}=0.000\right)$, los diagnósticos de colecistitis aguda $\left(\mathrm{X}^{2}=82.290\right.$, $\mathrm{p}=0.000)$ y colelitiasis $\left(\mathrm{X}^{2}=63.935, \mathrm{p}=0.000\right)$, y el motivo de conversión de inflamación severa (Test exacto de Fisher $[\mathrm{p}=0.000])$.

Tabla 2

Análisis multivariado de las variables consideradas predictores de la conversión de cirugía.

\begin{tabular}{llllllllll}
\hline & & & & & & & & \multicolumn{2}{l}{ IC 95\% } \\
\cline { 8 - 11 } & B & Error & Wald & gl & p & OR & & Inferior & Superior \\
\hline Constante & -7.063 & 1.166 & 36.663 & 1 & 0.000 & 0.001 & - & - \\
Edad & 0.037 & 0.018 & 4.319 & 1 & 0.038 & 1.038 & 1.002 & 1.075 \\
Sexo(a) & 1.832 & 0.596 & 9.461 & 1 & 0.002 & 6.246 & 1.944 & 20.071 \\
Colecistitis aguda(b) & 4.374 & 0.807 & 29.403 & 1 & 0.000 & 79.384 & 16.333 & 385.828 \\
\hline
\end{tabular}

Nota: (a) Masculino, (b) Si.

La regresión logística (tabla 2) muestra un buen ajuste del modelo a través de la prueba de Hosmer y Lemeshow ( $\mathrm{p}=0.738$ ), y una R2 de Nagelkerke de 0.608 en el paso 7 de los 8 ejecutados por el método Wald hacia atrás, evidenciando una predicción de conversión acertada del $92.9 \%$ de los casos. Las variables consideradas mejores factores predictivos de conversión de CL a CA, fueron: diagnóstico de colecistitis aguda $(\mathrm{OR}=79.384, \mathrm{p}=0.000)$, sexo $(\mathrm{OR}=6.246, \mathrm{p}=0.002)$ y edad $(\mathrm{OR}=1.038, \mathrm{p}=0.038)$.

\section{DISCUSIÓN}

Desde que en 1991 se iniciara la CL en el Perú, esta ha pasado más de 25 años ganándose el sitial que ocupa en la actualidad gracias a sus ventajas reconocidas a nivel mundial como son el menor dolor postoperatorio, cicatrización mínima, menor tiempo en el intrahospitalario y por ende, menores costos, además de la reinserción laboral rápida, comparándola con la CC (Ibáñez et al., 2007; Priego et al., 2008; Requena \& Gutiérrez, 2007; Serafini, 2016; Simón et al., 2006). Sin embargo, a pesar de su implementación masiva en los centros hospitalarios estatales y privados, no es de extrañar que exista la posibilidad de conversión a cirugía tradicional de manera inmediata y sin lugar a dudas, al presentarse alguna dificultad en la resolución del acto quirúrgico mediante la cirugía video asistida (Álvarez et al., 2013).

En el estudio, se encontró que la tasa de conversión se ubica dentro del rango esperado a nivel mundial y, especialmente, dentro los rangos establecidos a nivel latinoamericano que es de $0.8-20 \%$ (Álvarez et al., 2013; Bocanegra \& Córdova, 2013; Domínguez et al., 2011; Galloso et al., 2012; Ibáñez et al., 2007; Pekolj \& Sánchez, 2015; Priego et al., 2008; Serafini, 2016; Vargas et al., 2017), por diversas causas presentadas en cada caso.

La diferencia significativa de edades encontrada entre el grupo de pacientes a quienes no se realizó conversión a CA y el grupo a quienes sí se realizó, y los resultados de la regresión logística, confirman los reportes de diversos investigadores quienes afirman que la probabilidad de conversión aumenta con la edad (Bocanegra \& Córdova, 2013; Domínguez, Herrera, Rivera, \& Bermúdez, 2011; Domínguez, Rivera, et al., 2011; Galloso et al., 2012; Ibáñez et al., 2007; Priego et al., 2008; Serafini, 2016; Vargas et al., 2017), una 
de las razones que explicarían este comportamiento de los datos estaría relacionado con las diferentes patologías que las personas adquieren con el paso de los años las cuales aumentan su vulnerabilidad ante procedimientos invasivos como es el caso de una intervención quirúrgica.

Otro aspecto que deja confirmado el estudio, es que la tasa de conversión es más alta en varones que en mujeres; además, el modelo estadístico incluyó al sexo masculino como un predictor de la conversión de cirugía; hallazgo, que es de referencia común en los estudios (Álvarez et al., 2013; Bebko et al., 2011; Domínguez, Rivera, et al., 2011; Priego et al., 2008; Serafini, 2016). Una de las probables razones para explicar este hecho, podría estar ligada a factores de índole social o cultural que conllevan a realizar consultas tardíamente con el especialista.

Así mismo, se identificó un mayor porcentaje de conversión en las personas cuyo tipo de atención quirúrgica fue de urgencia, teniendo estrecha relación con el tipo de diagnóstico preoperatorio con el que ingresaron al servicio, ya que los pacientes con colecistitis aguda presentaron mayor índice de conversión que el resto de patologías vesiculares (Serafini, 2016). Por otro lado, el diagnóstico de colelitiasis tuvo el menor índice de conversión. Esto, indica la necesidad de educar a la población sobre la importancia de planificar la cirugía juntamente con el equipo de salud, de manera que la intervención se realice en las mejores condiciones físicas del paciente y disminuir el riesgo de conversión.

Entre los motivos de conversión a cirugía abierta destaca la inflamación severa del total de las personas sometidas a CL; las adherencias y el sangrado se presentaron en un porcentaje mínimo. Es importante considerar que la inflamación y edema del hilio hepático que acompañan a una colecistitis aguda grave, distorsionan la anatomía conductal y vascular, dificultando enormemente la disección de los tejidos (Álvarez et al., 2013), por lo que condiciona en gran medida al cirujano a decidir por la conversión a CA. Además, en una severa inflamación aguda o crónica, ocasionalmente el aspecto medial del infundíbulo está fusionado con la vía biliar, obliterando el triángulo de Calot y al persistir en esos casos de continuar con la técnica de CL, puede resultar no sólo en una lesión de la vía biliar, sino ocasionalmente en riesgo de vida por lesiones de las estructuras vasculares del ligamento hepatoduodenal (Visser, Parks, \& Garden, 2008).

Finalmente, es importante que los médicos consideren los factores predictivos de conversión como la colecistitis aguda, el sexo y la edad del paciente, para anticiparse a la necesidad de conversión de la cirugía. Así mismo, nunca debería considerarse la conversión a CA como un fracaso, pues esta es una decisión que lleva al cirujano a encontrar la mejor manera de enfrentar las dificultades en el momento oportuno antes de cometer iatrogenia (Arbues \& Bustos, 2016; Serafini, 2016; Vargas et al., 2017).

Los resultados mostraron como mejores factores predictivos de conversión de colecistectomía laparoscópica a cirugía abierta, al diagnóstico de colecistitis aguda, el sexo y la edad. La repetitividad de estos resultados en los diferentes estudios, sugiere la creación de un algoritmo en base a probabilidades que ayuden de forma temprana al cirujano en la programación del tipo de cirugía correspondiente.

\section{Declaración de financiamiento y de conflicto de intereses}

El estudio fue financiado por el autor, quien declara no tener algún tipo de conflicto de interés en la investigación realizada.

\section{Correspondencia}

Emilio Contreras Alomía

Clinica Ana Sthal, Iquitos, Perú.

e-mail: econtreras.alomia@gmail.com 


\section{REFERENCIAS BIBLIOGRÁFICAS}

Álvarez, L. F., Rivera, D., Esmeral, M. E., García, M. C., Toro, D. F., \& Rojas, O. L. (2013). Colecistectomía laparoscópica difícil, estrategias de manejo. Revista Colombiana de Cirugía, 28(3), 186-195. Recuperado de http://www.redalyc.org/articulo.oa?id=355535158002.

Arbues, G., \& Bustos, S. (2016). Colecistectomía dificultosa. In $87^{\circ}$ Congreso Argentino de Cirugía 2016 (Vol. 108, pp. 7-45). Buenos Aires: Asociación Argentina de Cirugía.

Balagué, C., Targarona, E. M., Ojuel, J., Viella, P., Espert, J. J., Pascual, S., ... Trias, M. (2000). Factores predictivos de conversión a cirugía abierta de la colecistectomía laparoscópica. Análisis de una serie prospectiva de 502 pacientes. Cirugía Española, 68(2), 139-143. https://doi. org/10.1056/NEJM199104183241601

Bebko, S., Arrate, E., Larrabure, L. I., Borda, G., Samalvides, F., \& Baracco, V. (2011). Eventos intraoperatorios inesperados y conversión en pacientes colecistectomizados por vía laparoscópica: Sexo maculino como factor de riesgo independiente. Revista de Gastroenterología Del Perú, 31(4), 335-344. Recuperado de http://sisbib.unmsm.edu.pe/bVrevistas/ gastro/vol31_n4/pdf/a06v31n4.pdf

Bocanegra, R. R., \& Córdova, M. E. (2013). Colecistectomía laparoscópica en el adulto mayor: complicaciones postoperatorias en mayores de 75 años en el Hospital Nacional Cayetano Heredia, Lima, Perú, del 2007 - 2011. Revista de Gastroenterología Del Perú, 33(2), 113120. Recuperado de http://www.scielo.org.pe/scielo. php?pid $=$ S1 $022-51292013000200003 \&$ script $=$ sci arttext

Borie, F., \& Millat, B. (2003). Encyclopédie médicochirurgicale. Techniques chirurgicales. Appareil digestif. Techniques Chirurgicales - Appareil Digestif, 40-950.

Bueno-Lledó, J., Planells-Roig, M., Sanahuja-Santafé, Á., García-Espinosa, R., Arnau-Bertomeu, C., \& Guillemot, M. (2005). Factores intraoperatorios predictivos del fracaso del régimen ambulatorio tras colecistectomía laparoscópica. Cirugía Española, 78(3), 168-174. https://doi.org/10.1016/S0009-739X(05)70911-8

Bueno, J., Serralta, A., Planells, M., Pous, S., Ballester, C., Ibáñez, F., \& Rodero, D. (2001). Colecistectomía laparoscópica y sus complicaciones: nuestra experiencia en nueve años. Cirugía Española, 69(5), 467-472. https://doi.org/10.1016/S0009-739X(01)71784-8

Campos, S. F., \& Lara, J. L. (2012). Colelitiasis y coledocolitiasis. In C. Gutiérrez S \& C. Arrubarrena A (Eds.), Fisiopatología quirúrgica del aparato digestivo (4a ed., pp. 378-396). México: Manual Moderno.

Domínguez, L., Herrera, W. E., Rivera, A. M., \& Bermúdez, C. E. (2011). Colecistectomía de urgencia por laparoscopía por colecistitis aguda en adultos mayores. Revista Colombiana de Cirugía, 26(2), 93-100. Recuperado de http://www.redalyc.org/articulo.oa?id=355534499003.
Domínguez, L., Rivera, A., Bermúdez, C., \& Herrera, W. (2011). Análisis de los factores de conversión durante colecistectomía laparoscópica a abierta en una cohorte prospectiva de 703 pacientes con colecistitis aguda. Cirugía Española, 89(5), 300-306. https://doi. org/10.1016/j.ciresp.2011.01.009.

Galloso, G. L., Frías, R. A., Pérez, O., Petersson, M., \& Benavides, S. (2012). Factores que influyen en la conversión de la colecistectomía video laparoscópica a cirugía tradicional. Revista Cubana de Medicina Militar, 41(4), 352-360. Recuperado de http://scielo.sld.cu/scielo.php?script=sci arttext\&pid=S0138-65572012000400005.

Ibáñez, L., Escalona, A., Devaud, N., Montero, P., Ramirez, E., Pimentel, F., ... Guzmán, S. (2007). Colecistectomía laparoscópica: experiencia de 10 años en la Pontificia Universidad Católica de Chile. Revista Chilena de Cirugía, 59(1), 10-15. https://doi.org/10.4067/S071840262007000100004.

Lucema, J. R., Coronel, P., \& Orellana, Y. (2008). Colecistectomía laparoscópica en colecistitis aguda. Factores de riesgo para la conversión a cirugía abierta. Revista Electrónica de Biomedicina, 2. Recuperado de http://biomed.uninet.edu/2008/n2/lucena.html.

Paredes, J. P., Puñal, J. A., Beiras, A., Barreiro, F., Segade, F. R., Martínez, J., ... Potele, J. (2001). 1.000 colecistectomías laparoscópicas: indicaciones y resultados. Cirugía Española, 70(4), 195-199. https:// doi.org/10.1016/S0009-739X(01)71877-5.

Pekolj, J., \& Mazza, O. (2005). Patología biliar aguda. In S. Perera \& H. García (Eds.), Aparto digestivo: Cirugía de Urgencia (2a ed., pp. 557-571). México: Editorial Médica Panamericana.

Pekolj, J., \& Sánchez, R. (2015). Complicaciones de la cirugía abdominal Sung Ho Hyon. In J. Pekolj, V. Ardiles, \& S. H. Hyon (Eds.), Complicaciones más frecuentes de la cirugía biliar (1a ed., pp. 467-518). Buenos Aires, Argentina: Delhospital ediciones. Recuperado de https:// www1.hospitalitaliano.org.ar/multimedia/archivos/ noticias_archivos/13/Notas_PDF/13_Complic_cirugia corte.pdf.

Priego, P., Ramiro, C., Molina, J. M., Rodríguez, G., Pina, J. D., Lobo, E., ... Fresneda, V. (2008). Factores asociados a la conversión en la colecistectomía laparoscópica. SECLA Endosurgery, 25. Recuperado de http://www. seclaendosurgery.com/seclan25/articulos/art03.htm.

Ramos, A. E., Álvarez, Á. C., Lorenzo, A., Vallés, M. E., \& Gallardo, R. L. (2014). Factores determinantes de las conversiones en la colecistectomía laparoscópica. Correo Científico Médico, 18(4), 611-622. Recuperado de http://scielo.sld.cu/scielo.php?script=sci_ arttext\&pid=S1560-43812014000400003. 
Requena, A., \& Gutiérrez, Y. (2007). Causas de conversión en colecistectomía laparoscópica caja nacional de salud H. O Nro. 22000 - 2005 Cochabamba. Gaceta Médica Boliviana, 30(2), 38-41. Recuperado de http://www.scielo.org.bo/scielo.php?script=sci_ arttext\&pid=S1012-29662007000200008.

Sepulveda, A. (1991). Libro de reporte operatorio. Lima, Perú.

Serafini, V. H. (2016). Formación en cirugía abierta en la era videoscópica: ¿Es necesario seguir enseñando cirugía abierta? In $87^{\circ}$ Congreso Argentino de Cirugía 2016 (Vol. 108, pp. 66-202). Buenos Aires: Asociación Argentina de Cirugía.

Simón,L.,Sánchez,C.A.,Acosta,L.R.,\&Sosa,R.(2006).Costes: colecistectomía laparoscópica frente a colecistectomía convencional. Revista Cubana de Cirugía., 45(3-4). Recuperado de http://scielo.sld.cu/scielo.php?script=sci arttext\&pid=S0034-74932006000300005.
Vargas, L. J., Agudelo, M. B., Lizcano, R. A., Martínez, Y. M., Velandia, E. L., Sáchez, S. J., ... Quintero, M. J. (2017). Factores asociados con la conversión de la colecistectomía laparoscópica a colecistectomía abierta. Revista Colombiana de Gastroenterología, 32(1), 20-23. https://doi.org/10.22516/25007440.125.

Visser, B. C., Parks, R. W., \& Garden, O. J. (2008). Open cholecystectomy in the laparoendoscopic era. The American Journal of Surgery, 195(1), 108-114. https:// doi.org/10.1016/j.amjsurg.2007.04.008

Recibido: 13/10/2016 Aceptado: 29/11/2016 